\title{
Adaptive Spectrum Opportunity Access in Cognitive Radio Networks
}

\author{
Mahdi Pirmoradian, Student Member IEEE; Olayinka Adigun, Student Member IEEE; Christos Politis, Senior Member IEEE \\ WMN Research Group, Kingston University \\ London, UK \\ \{m.pirmoradian, o.adigun, c.politis\}@kingston.ac.uk
}

\begin{abstract}
This paper presents the Adaptive Spectrum Opportunity Access (ASOA) scheme. The proposed scheme greatly improves spectrum utilization and provides improved QoS for the secondary users. The secondary data delivery time is adapted in different spectrum opportunities through ASOA scheme based on the estimated collision rates in a sophisticated way. The performance evaluations in terms of the channel utilization, channel collision, secondary data delivery and handoff rates are assessed and compared with the Maximum Idle Channel Remaining Lifetime (MICRL) schemes. The simulation results confirm the viability of the ASOA scheme in comparison with MICRL schemes.
\end{abstract}

Keywords - Channel collision, Cognitive radio, Spectrum decision, Spectrum handoff

\section{INTRODUCTION}

Spectrum has become a scarce radio resource in emerging wireless technologies due to increasing number and varieties of intelligent wireless link applications. Mobile networks are expected to be congested by more users and applications. Expectations in the rising volume of data transmitted over mobile networks which is estimated to be doubled every year, needs more radio spectrum demand in the forthcoming years [1]. In some cases, operators estimate data consumption will increase ten times in the next five years [2]. An estimation from ITU-R shows the extra spectrum demand in 2020 will be $1280-1720 \mathrm{MHz}$ [3] to supplement the current allocated radio spectrum in mobile networks. Investigations from Spectrum Policy Task Force (SPTF) also show that $85 \%$ of current allocated radio frequency bands are either partially or completely unused at different given times and geographical areas [4]. Also, an investigation of spectrum measurements in different regions of Europe denotes that spectrum utilization on $400 \mathrm{MHz}$ to $3 \mathrm{GHz}$ bands is less than $11 \%$ [5]. For these reasons, experts are interested to explore new spectrum utilization techniques and spectrum allocation concepts which will tackle critical challenges in spectrum allocation policies, spectrum trading techniques and spectrum management concepts as well as on technological ground. With all the envisage challenges in mind, spectrum sharing concept will be a great solution to tackle radio spectrum scarcity in the near future and enable wireless devices reach their full operational capacity. This means license-exempt users (also referred to secondary users or cognitive users) can use that open licensed spectrum bands without disrupting the licensed transmission.

Various spectrum-sharing models such as open sharing hierarchical access and dynamic exclusive usage models are studied in [6].

Two prominent spectrum-sharing schemes within cognitive radio context, targeted between licensed users and licenseexempt users are: Overlay Spectrum Access, also known as Opportunistic Spectrum Access (OSA) and Underlay Spectrum Access. OSA mechanism was first presented by Mitola under Cognitive Radio (CR) concept and later developed by many researchers. This new technology has the potential to dramatically increase radio spectrum utilization by allowing secondary usage of unused licensed spectrums in an interference-limiting manner.

Cognitive radio is a paradigm for next generation wireless communications, which is seen as the solution to the current low utilization of the radio spectrum. According to the CR's definitions [7], [8], CR has the capability of being cognitive, reconfigurable and self-organized to fulfill the functions of spectrum sensing, spectrum decision, spectrum sharing and spectrum mobility under Dynamic Spectrum Access (DSA) mechanism. DSA capability allows cognitive device to monitor available licensed channels and adapts its transceiver parameters to the new radio state in a dynamic manner. Spectrum decision is a major decisive process in seeking appropriate spectrum hole in cognitive cycle. Therefore, this process needs to be further developed and can be implemented on MAC layer with correlation links to the PHY layer. As explained earlier, cognitive radio responses to observe and exploit interim spectrum opportunities (spectrum holes or white spaces) in specific period of time without causing interference to the licensed receivers. Thus, a CR system will be able to coexist with heterogeneous wireless networks.

This study presents an adaptive spectrum selection scheme based on the average channel collision rate in specific period of time, meaning that the secondary transmission time would be adapted with respect to the channel characteristics and radio environment parameters in different spectrum holes. The spectrum decision framework would be evaluated in a multi-licensed channels network based on the central decision approach. 
The rest of the paper is structured as follows. In section II, related work is illustrated. In section III, system topology and network parameters are illuminated. Section IV introduces the analytical model and different spectrum decision schemes. Simulation results and performance evaluations are represented in section $\mathrm{V}$, finally the paper concluded in section VI.

\section{RELATED WORK}

We reviewed most relevant topics to the present paper that have been studied by researchers. The modelling of spectrum handoff and primary user's behaviours can be classified into the following five types: (1) the two-state Markov chain [9], [10], [11]; (2) the arbitrary ON/OFF random process [12] [13] [14]; (3) the Bernoulli random process [15]; (4) the birth death process with multidimensional Markov chain [16]; and (5) the PRP M/G/1 queuing model [17] [18]. In [19], authors present an opportunistic spectrum access scheme based on the channel idle probability. A spectrum hole prediction model based on the IEEE 802.11 standard is presented in [20]. The scenario presents a multi-user networks, where distribution of interval time between two consecutive packets are considered as prominent channel selection condition. Also [21], presented a dynamic channel selection scheme for agile low power wireless packet switched networks over unlicensed bands with application on short-range wireless communication devices. Furthermore, [22] proposed a Channel Selection Scheme based on Minimum Collision Rate Algorithm (CSS-MCRA) and the channel selection scheme based on Minimum Handoff Rate Algorithm (CSSMHRA) under the constraint that the collision probability is bounded below collision tolerable level. In [23], spectrum handoff mechanism based on transition probabilities of licensed channels' states are presented. In the aforementioned works, licensed channels are modeled by binary ON/OFF signal. Also the authors in [9]-[12] assumed that the primary usage of the channels have the Markov property. It was assumed that the secondary user can estimate the distributions of the $\mathrm{ON}$ period and the $\mathrm{OFF}$ period based on long-term observations. Basically in cognitive radio networks, QoS of the SUs and PUs will be affected by spectrum handoff and channel selection techniques, which directly increase secondary delivery time and power consumption.

According to the literature, both the PU arrival time and channel selection strategy on the SU side significantly influence secondary data delivery over licensed networks. This paper focuses on OSA approach, where secondary users' desire access to unoccupied available spectrum bands with respect to the instantaneous spectrum sensing information. The scheme could be run at the SU side, which responses to protect PU against harmful collision. Also, based on the channel characteristics and predefined collision rates, secondary transmission time will be adapted in different channels. The performance of the proposed spectrum decision scheme will be compared with Maximum Idle Channel Remaining Lifetime (MICRL) schemes. In both mechanisms, spectrum handoff during secondary delivery over licensed channels and successful data delivery are considered as the main performance evaluation metrics. The proposed mechanisms and analytical procedures will be explained in the next sections.

\section{SYSTEM MODEL AND CONCEPTS}

We consider an open licensed spectrum network with static wireless nodes communicating with each other using $\mathrm{N}$ licensed channels $\left\{c h_{1}, c h_{2}, \ldots c h_{N}\right\}$, whilst a cognitive radio network is located within the licensed coverage area. The primary users have high priority of using the licensed channels. The secondary users are assumed to be static and desire to utilize available licensed channels in an opportunistic manner based on their cognitive functionalities. The cognitive nodes are equipped with spectrum sensor devices that monitor and report channel states to the central node via dedicated channels. Also, the sensors outcome can be defined as a sequence of binary signal $\{0,1\}$, which represents the vacancy and occupancy of observed channels at time instance t. In the assumed topology, the central node informs secondary users for sensing available channels by sending Sensing Flag (SF) signal in a proactive manner. In this paper, we assume that the duration of idle and busy time is much greater than sensing time. The network notations and some defined parameters are explained in table 1.

\section{Table 1: Network notations}

\begin{tabular}{|c|l|}
\hline $\mathbb{N}(t)$ & Set of vacant channels at time $\mathrm{t}$ \\
\hline$X_{j}$ & Spectrum hole on channel $\mathrm{j}$ \\
\hline$\mu_{o f f}^{i}$ & $\begin{array}{l}\text { Parameter of Exponential distribution } \\
\text { for idle period on channel } i\end{array}$ \\
\hline$\mu_{o n}^{i}$ & $\begin{array}{l}\text { Parameter of Exponential distribution } \\
\text { for busy period on channel } i\end{array}$ \\
\hline$f_{X}(x)$ & $\begin{array}{l}\text { Probability density function of idle } \\
\text { channel }\end{array}$ \\
\hline$E()$. & Average expected function \\
\hline$S H_{j}(t)$ & $\begin{array}{l}\text { Selected spectrum hole on channel } \mathrm{j} \text { at } \\
\text { time } \mathrm{t}\end{array}$ \\
\hline $\bar{F}(t)$ & Survival function of idle channel \\
\hline
\end{tabular}

\section{A. Licensed Channel Usage}

Surveying, analysing, processing and predicting of interim unused spectrum bands in both frequency and time domains require highly computational mathematical techniques. We assume utilisation of the licensed bands is modelled as a Poisson process with arrival rate parameter $\mu$, therefore, the number of events in time interval $(t, t+\tau]$ can be given by [24]; 


$$
P[(N(t+\tau)-N(t))=k]=\frac{(\lambda \tau)^{k} e^{-\lambda \tau}}{k !} \quad k=0,1,2, \ldots
$$

Here $N(t+\tau)-N(t)$ is the number of events in time interval $(t, t+\tau]$. A single duration of utilization of the licensed band by a PU is denoted by $T_{\text {on }}(\mathrm{ON}$ state or Busy time) and a single duration of the licensed band being idle is denoted by $T_{\text {off }}$ (OFF state). The duration between two utilization periods (inter-arrival rate of the PU) are identical independent distribution (i.i.d) random variables, with exponential distribution. It is assumed that the arrival rates and probability density functions that can be estimated by the existing methods [25] are known to the SUs. Consequently, the probability density function of OFF state in the licensed band can be expressed as:

$$
f_{T Z}\left(\mathrm{t}, \mu_{\mathrm{Z}}\right)=\mu_{\mathrm{Z}} \mathrm{e}^{-\mu_{\mathrm{Z}} \mathrm{t}} \quad t \geq 0
$$

Where $\mathrm{Z}$ can be defined as OFF or ON states. Also, $\boldsymbol{\mu}$ denotes OFF and ON arrival rates. For simplicity, in the rest of the article OFF and ON states replace by $\mathrm{X}$ and $\mathrm{Y}$ random variables respectively (see Fig.1).

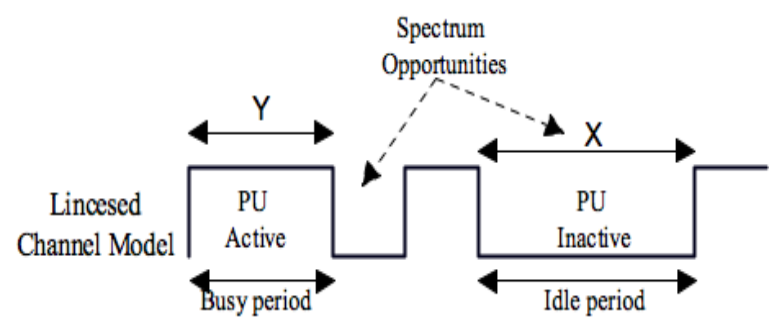

Figure 1: Primary user channel model; $\mathrm{X}$ and $\mathrm{Y}$ are identical independent random variables.

\section{B. Preliminary of Adaptive Spectrum Opportunity Access}

This section illustrates the adaptive spectrum decision scheme. The main aim of the scheme is to seek and allocate appropriate unoccupied spectrum bands with respect to the channel collision and channel handoff rate constraints. The proposed decision procedure runs into two steps (sensing and decision steps) respectively.

Step1. Sensing (at SU)

$S F \leftarrow 1$

$S U_{k}$ observes $c h_{1}, c h_{2}, \ldots, c h_{N}$

Reports

$S_{\mathcal{M}}^{k}=\left\{S_{1}^{k}, S_{2}^{k}, \ldots, S_{N}^{k}\right\}$ where $S_{1, \ldots, N}^{k} \in\{0,1\}$ and $\mathcal{M}=$

$1, \ldots, N$

End

Step2. Decision (at the CN)

Evaluate transmission times

If

$S_{o p}^{k}=1$,

establish current channel and transmission time to $S U_{k}$ Else
Select appropriate channel using maximum transmission time

Establish $c h_{o p}$ and $T_{o p}$ to the $S U_{k}$

End

The target-operating channel is selected through the procedure above. The ASOA scheme and its flowchart are exhibited in figure 2.

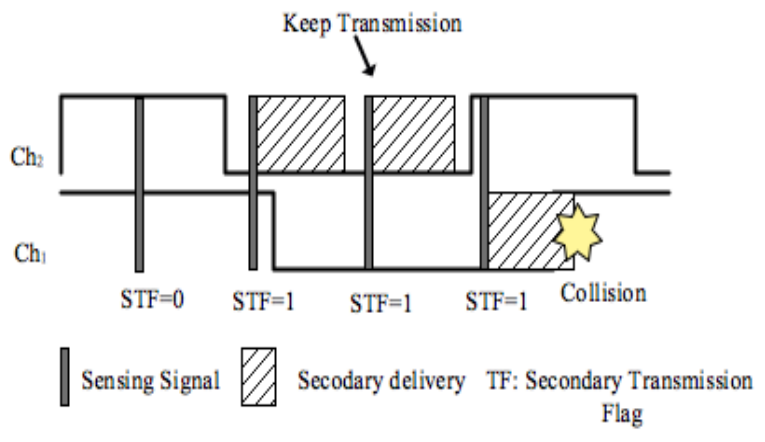

Figure 2. a) Illustration and concept of ASOA scheme

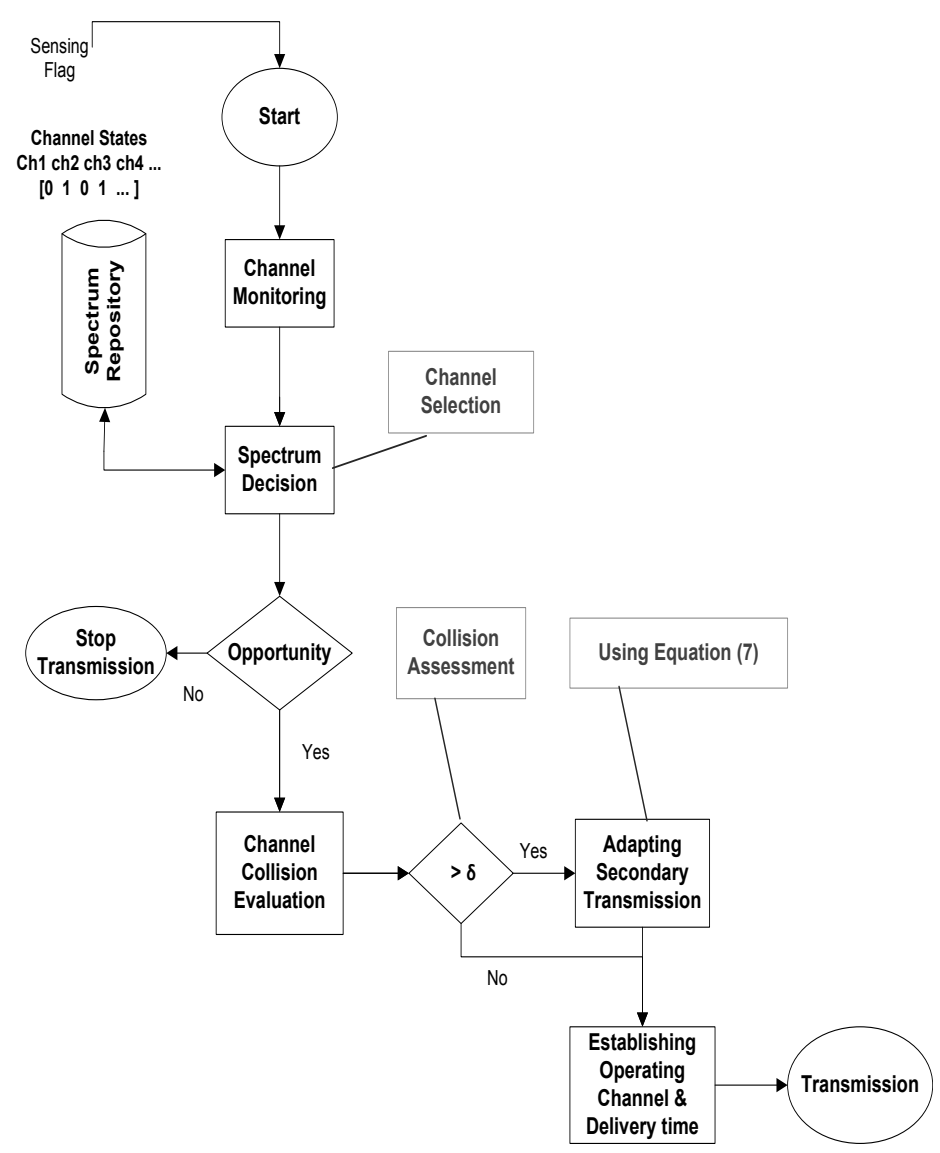

Figure 2. b) The flowcharts of establishing operating channel and transmission time to a typical SU. 


\section{ANALYTICAL MODEL}

\section{A. Channel Utilization and Collision Rate}

As discussed earlier, the $\mathrm{ON}$ and OFF channel states are modeled by i.i.d exponentially random variables. Generally, according to the nature of the licensed channel model and characteristics of the random variables, the average secondary use of the established channel can be written as [26];

$$
\mathbb{E}=\mathrm{T}_{s} \int_{\mathrm{T}_{s}}^{\infty} f_{X}(x) d x+\int_{0}^{\mathrm{T}_{s}} x f_{X}(x) d x
$$

Where the average channel utilization through specific period of time $\mathrm{T}$ can be defined as,

$$
U_{i} \%=\frac{\mathbb{E} \cdot \sum_{n=0}^{N-1} \Omega_{i}\left(t_{n}\right)}{T} \approx \frac{m \mathbb{E}}{n T_{x}} \times 100 \%
$$

Here $\Omega_{i}\left(t_{n}\right) \in\{0,1\}$ can be defined as channel selection outcomes, where 1 represents channel $\mathrm{i}$ is selected as operating channel. We assume all channels are sensed frequently, therefore, $m, n$ and $T_{x}$ represent number of channel selection, number of sensing and interval sensing time in channel $\mathrm{i}$, in the period of time $\mathrm{T}$ respectively. AlsoT $_{s}$, denotes secondary activity time in an interim spectrum hole obtained from radio characteristics. The first item in the right hand side of equation (3) represents probability of successful transmission and the second item introduces the mean value of either unsuccessful secondary data transmission or collision. Based on that, the Collision Rate $(\mathcal{C R})$ of the secondary user $\mathrm{k}$ in channel $\mathrm{i}$ can be defined as;

$$
\mathcal{C} \mathcal{R}_{k}^{i}=\frac{\int_{0}^{T_{s}^{i}} x f_{X}(x) d x}{\mathbb{E}^{i}(\mathrm{X})+\mathbb{E}^{i}(\mathrm{Y})}
$$

Clearly, the secondary transmission time in channel i can be obtained by.

$$
\begin{gathered}
T_{s}^{i} \leq\left[\delta^{i}\left(\mathbb{E}^{i}(X)+\mathbb{E}^{i}(Y)\right) \cdot \mathbb{E}^{i}(X)\right]^{1 / 2} \\
T_{s}^{i} \in\left\{T_{\text {smin }}^{i}\left[\delta^{i}\left(\mathbb{E}^{i}(X)+\mathbb{E}^{i}(Y)\right) \cdot \mathbb{E}^{i}(X)\right]^{\frac{1}{2}}\right\}
\end{gathered}
$$

\section{Proof. See Apendix1}

Where $\delta^{i}, T_{s}^{i}, T_{s \min }^{i}$ are collision rate constraint, secondary transmission's time, and minimum required secondary delivery time in channel $i$.

Channel collision represents the amount of secondary and primary transmission overlapping time in the same channel. In fact, the metric clearly reveals efficiency of the proposed spectrum handoff techniques.

From (3), average channel collision in channel $\mathrm{i}$ in specific period of time $\mathrm{T}$ can be defined by,

$$
A C C_{k}^{i} \%=\lim _{T \rightarrow \infty} \frac{\sum_{k=1}^{i^{i}} \text { Expected collision in }[0, T]}{T} \times 100 \%
$$

Where $l{ }^{i}$ represents the number of collision during $\mathrm{T}$ second in channel $i$. Generally, average channel collision with regards to (8) can be given by,

$$
\begin{gathered}
\mathbb{E} \mathcal{C}^{i}=\left[\mathbb{E}^{i}(\mathrm{X})-\left(T_{s}^{i}+\mathbb{E}^{i}(\mathrm{X})\right) e^{-\mu_{o f f}^{i} T_{s}^{i}}\right] \\
T A C C_{k}^{i} \%=\lim _{T \rightarrow \infty} \frac{\mathbb{E C ^ { i } \cdot \sum _ { k = 1 } ^ { l ^ { i } } \mathbb { C F } _ { i } ( t _ { n } )}}{T} \times 100 \%
\end{gathered}
$$

Where $l^{i}, \mathbb{C F}_{i}\left(t_{n}\right)$ represent the number of channel collision during $\mathrm{T}$ and collision flag in channel $\mathrm{i}$ at sensing time $t_{n}$. Due to the frequent sensing mechanism TACC will reach its peak value when $\frac{l \mathbb{E} \mathcal{C}}{n T_{x}} \approx \frac{\mathbb{E} \mathcal{C}}{T_{x}}$ in channel $i$.

\section{B. Success Data Transmission Time}

This section provides further information in secondary data delivery estimation during specific period of time T. Thus, based on the sensing outcomes and channel characteristics, the effective secondary transmission time for secondary user $\mathrm{k}$, over $\mathrm{N}$ licensed channels can be yielded from (3) as,

$$
\begin{gathered}
S D T T_{k}=\sum_{i=1}^{N} \sum_{j=1}^{M} T_{s}^{i j} \exp \left(-\mu_{o f f}^{i} T_{s}^{i j}\right)-\sum_{i=1}^{N} \sum_{k=1}^{l(i)}\left[\mathbb{E}^{i}(\mathrm{X})-\right. \\
\left.\left(T_{s}^{i}+\mathbb{E}^{i}(\mathrm{X})\right) e^{-\mu_{o f f}^{i} T_{s}^{i}}\right]
\end{gathered}
$$

Where $\mathrm{M}$ denotes number of secondary access during $\mathrm{T}$ second in channel i. Generally, total secondary transmission is expected to reach its peak when $\left(\mathcal{N}_{S T}-\mathcal{N}_{C}\right) T_{s} \exp \left(-\mu_{o f f} T_{s}\right)$, when secondary transmission time slot is assumed to be fixed. Assuming that channel data rates is $\mathcal{R}$ bit/s, therefore, secondary data delivery can be estimated by $\mathcal{R} .\left(\mathcal{N}_{S T}-\mathcal{N}_{C}\right) T_{S} \exp \left(-\mu_{\text {off }} T_{S}\right)$ bits. In the equations above, $\mathcal{N}_{S T}, \mathcal{N}_{C}$ represent number of secondary transmission and collision in $\mathrm{T}$ second respectively.

\section{Channel Lifetime Expectation}

This subsection provides a brief explanation on the estimation of remaining idle channel lifetime based on the alternative renewal theory [27]. We assume the licensed channel model follows a two state repairable system with i.i.d random variable states ( $X$ and $Y$ as in figure 1). In this system, the functioning period and the system down time for the repair period are random variables. Hence the sequence of random variables $\left\{Y_{i}+X_{i} \mid i=1,2, \ldots\right\}$ are mutually independent and identically distributed. Thus the remaining idle channel lifetime at sensing time instance $t$ in channel $i$ can be obtained as [24],

$$
E\left(Z^{i}(t)\right)=\frac{\mu_{o n}^{i}+\mu_{o f f}^{i}}{\mu_{o n}^{i} \mu_{o f f}^{i}}-t \cdot \exp \left[-\left(\mu_{o n}^{i}+\mu_{o f f}^{i}\right) t\right]
$$

Where $\mathrm{Z}$ and $\mathrm{t}$ represent idle channel lifetime and sensing time instance. 
V.

\section{SPECTRUM DECISION SCHEMES}

\section{A. Adaptive Spectrum Opportunity Access scheme}

The main goal of this technique is to explore and select appropriate idle channels based on the secondary data delivery time. In principle, the suitable white space would be selected through ASA decision process. Hence, the channel access scheme can be expressed as;

$$
\begin{aligned}
& S_{j}^{A S O A}(t)=\operatorname{argmax}_{i \in \mathbb{N}(t) \& 0 \leq i \leq N}\left(T_{s}^{i}\right) \\
& \text { st. } \quad \frac{\mathbb{E}^{i}(X)-\left(T_{s}^{i}+\mathbb{E}^{i}(X)\right) \exp \left(-\mathbb{E}^{i}(X) T_{s}^{i}\right)}{\mathbb{E}^{i}(X)+\mathbb{E}^{i}(Y)} \leq \delta
\end{aligned}
$$

Where SU selects appropriate channel and continues its transmission as long as operating channel is being vacant. However, spectrum handoff occurs whenever a PU appears in the frequency band. At this point secondary data delivery will be continued in new operating channel under specific secondary transmission time. Obviously, this technique will degrade channel collision and handoff rates as well as energy consumption, which are indicated in next section.

\section{B. Maximum Idle Channel Remaining Lifetime (MICRL) scheme}

MICRL scheme has been studied in previous researches and considered as an appropriate spectrum hole selection scheme in dynamic systems. The main goal of this scheme is to enhance secondary data delivery and decrease spectrum handoff as much as possible by selecting reliable spectrum hole. This scheme estimates the remaining lifetime of the unoccupied licensed channels using channels' characteristics, and so the channel with maximum remaining lifetime is allocated to the SU for data delivery. This spectrum decision strategy can be expressed as;

$$
\left.S H_{j}^{M I C R L}(t)=\operatorname{argmax}\left\{i \mid E\left(Z^{i}(t)\right)\right\} i \in \mathbb{N}(t)\right\}
$$

\section{NUMERICAL RESULTS}

In this section, some numerical results are presented to demonstrate the performance of the proposed ASOA scheme using specific network parameters. The simulation results are representative of the considered network. The main goal in the simulation is to obtain channel utilization, channel collision rate, spectrum handoff rate and secondary data delivery in the licensed channels for the interim spectrum holes. Hence, the proposed ASOA scheme performance is compared with MICRL scheme. The licensed network includes six-licensed channels, having different channel characteristics $1 / \mu_{o f f}$ and $1 / \mu_{o n}$, and initializing to $\{3,1,5,8,4,5\}$ and $\{2,2,2,1,3,4\}$ second respectively. Also, all available channels are to be sensed in a reactive manner. The evaluations are achieved in specific time slot of 2000s. Hence, we assume switching latency among channels is neglected and spectrum handoff is perfect. The assumptions are taken into equations (13) and (14) for ASOA and MICRL schemes. The results are exhibited in the following figures.

Figure 3 shows average secondary utilization of the 6 licensed channels for ASOA and MICRL schemes. The results show that MICRL scheme mainly targets licensed channel 4, while ASOA targets channels 3, 4, 5, and 6 for data transmission. It is evident that channel 4 uses majority of the interim spectrum holes for secondary transmission using the MICRL scheme. The utilization peaks at 0.33 in channel 4 for the MICRL scheme, while channel utilization for ASOA varies between 0.05 to 0.19 in channels 3, 4, 5, and 6 . The adaptive property of ASOA has enabled it to have better secondary users' utilization of the spectrum holes in all channels when compared to MICRL, whose secondary utilization is mainly in channels, which has a considerably longer spectrum hole duration.

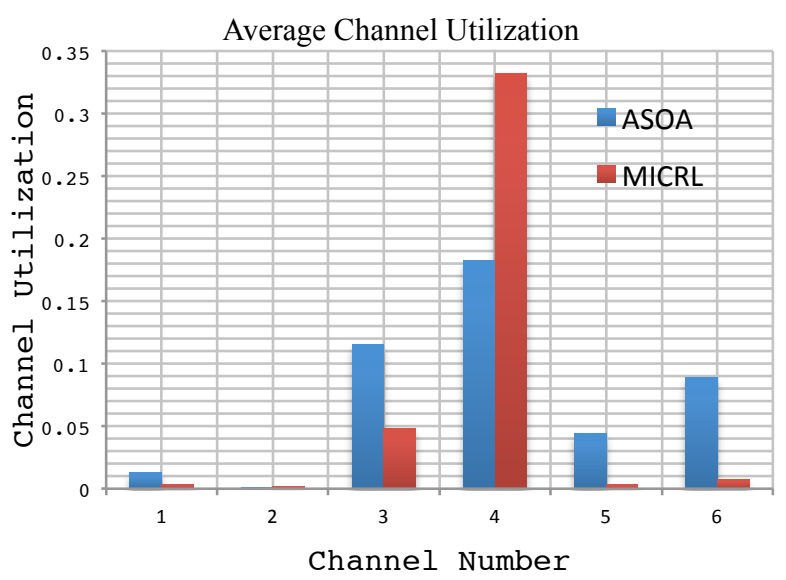

Figure 3. Average channel utilization by secondary user

High collision level undermines QoS of the licensed and license-exempt users in spectrum sharing area. Therefore collision level is considered to evaluate the proposed ASOA scheme. Figure 4 shows the average channel collision for ASOA and MICRL scheme in the 6 licensed channel. It can be seen for channel 4 that MICRL has a higher collision rate of 0.01 while ASOA has a reduced collision rate of 0.004 . With regards to the channels on and off characteristics and the secondary channel utilization shown in figure 3 , the higher collision levels observed in channels 1, 3, 5 and 6 for the ASOA schemes can be attributed to the fact that the ASOA schemes has a higher secondary channel utilization in these channels when compared to MICRL with reduced utilization. The absence of collision for the ASOA scheme in channel 2 also shows the superior collision avoidance ability of ASOA schemes when compared to MICRL. It is evident that ASOA scheme adapts its secondary utilization transmission time in order to degrade harmful disruption to the licensed transmissions and this will be useful in keeping channel collision levels below predefined values. 


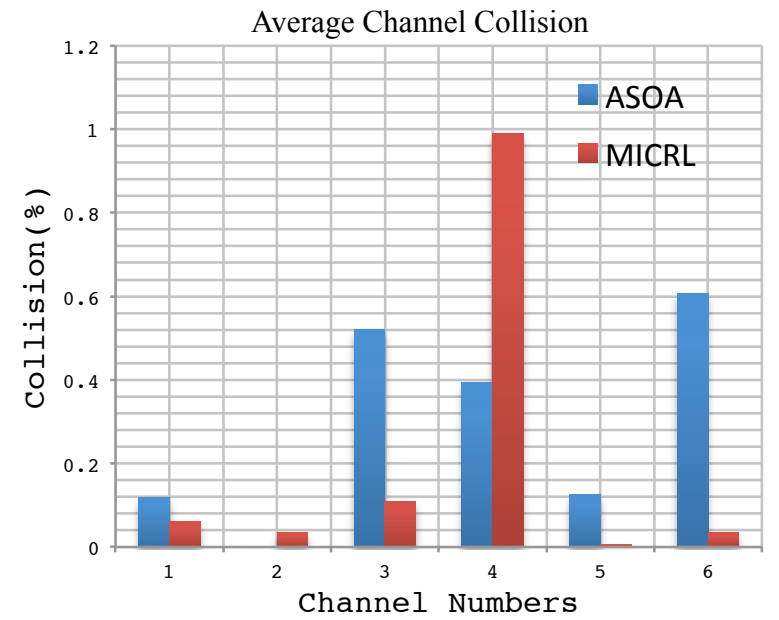

Figure 4. Average channel collision in 6 licensed channels

Figure 5 presents the result of the successful secondary data delivery performanc metric for ASOA and MICRL in the assumed network. We evaluate the successful secondary data delivery as a prominent performance metric in the assumed network as this greatly affects the QoS for the secondary users. The figure shows the secondary data delivery for the 6 licensed channels during a simulation period of $10000 \mathrm{~s}$. The capacity of licensed channels is assumed to be $2 \mathrm{Mb} / \mathrm{s}$. The results show that ASOA scheme has a higher level of successful secondary data delivery throughout the duration of the simulation. ASOA scheme has an average of $43 \mathrm{MB}$ while MICRL scheme has an average of $38 \mathrm{MB}$. This clearly shows that ASOA scheme performs secondary transmission with regards to the collision constriant necessary to fullfil the QoS requirement.

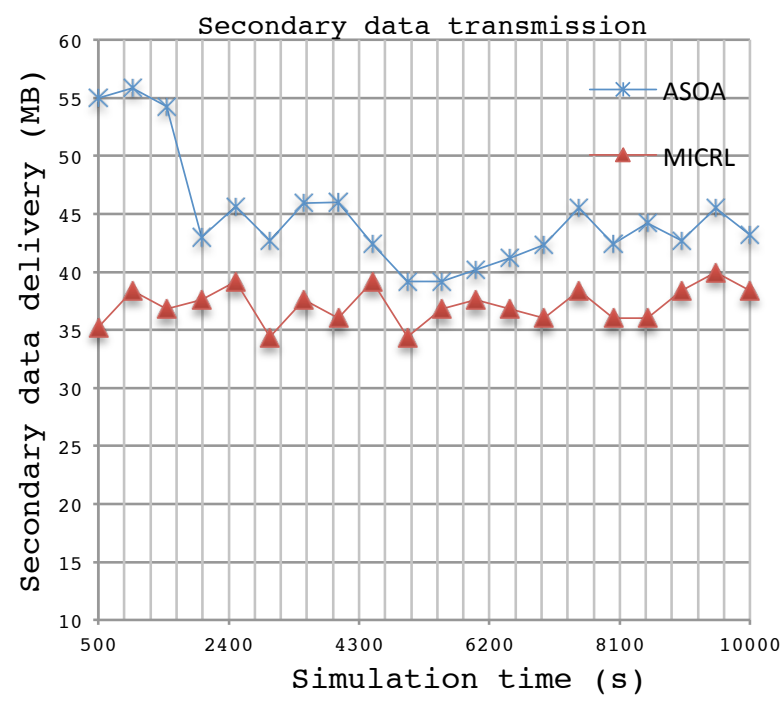

Figure 5. Successful data delivery over 6 licensed channels in $10000 \mathrm{~s}$.

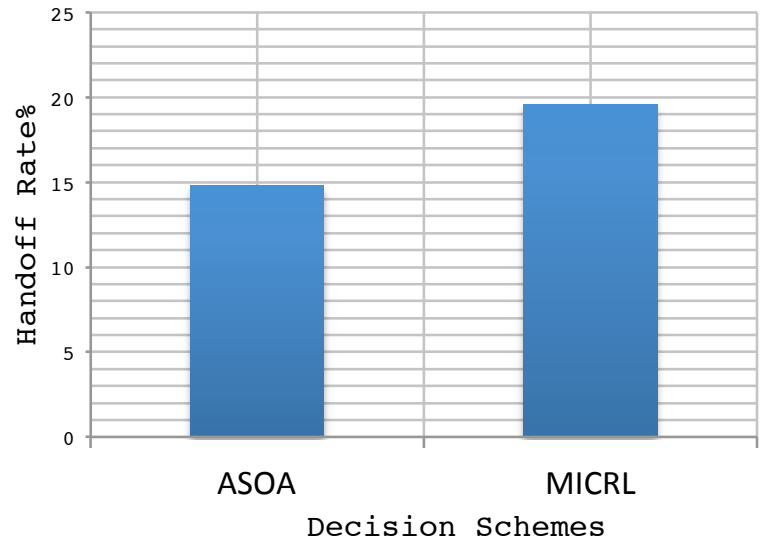

Figure 6. Successful data delivery over 6 licensed channels in 10000 s.

The frequency handoff is evaluated for the proposed schemes and the results are shown in figure 6 . The spectrum handoff is shown to improve for the ASOA scheme when compared with the MICRL scheme. The simulation outcomes demonstrates that handoff rates peaks at $14.8 \%$ and $19.6 \%$ for ASOA and MICRL schemes respectively. The reduced frequency handoff rate shown in ASOA coupled with it higher successful secondary data delivery will help improve the QoS. The evaluation of channel collision control is shown in figure 7 and 8 over 6 channels. Figure 7 shows the channel collision control for channels 1,2,3 while figure 8 shows the channel collision control for channels 4,5,6. Despite the fact that collision level tend to increase with the increase in secondary utilization, the graphs confirm that ASOA scheme adapts its transmission time to keep channel collision rate below 0.01 (constriant level) in all licensed channels.

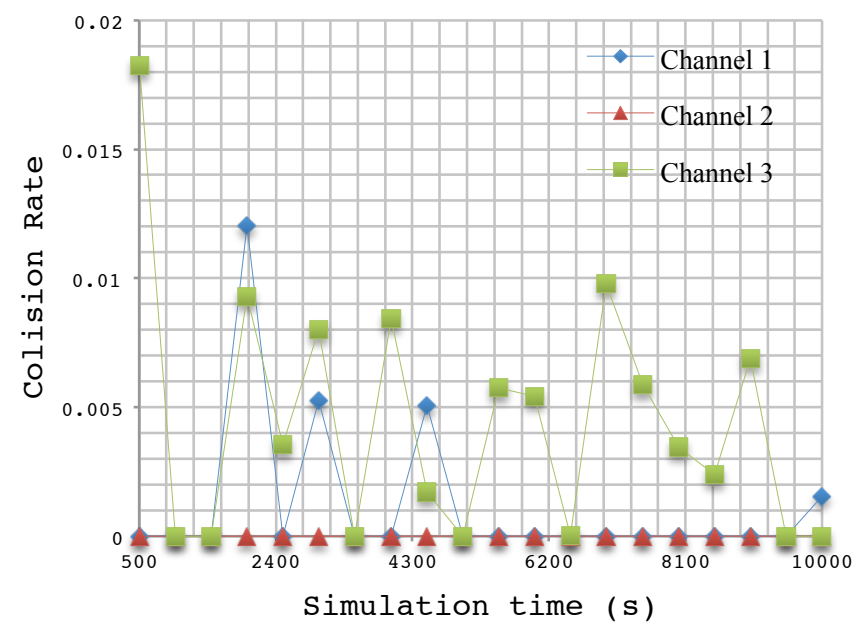

Figure 7. Average channel collision control in licensed channels 1, 2, 3 during 10000s for ASOA scheme 


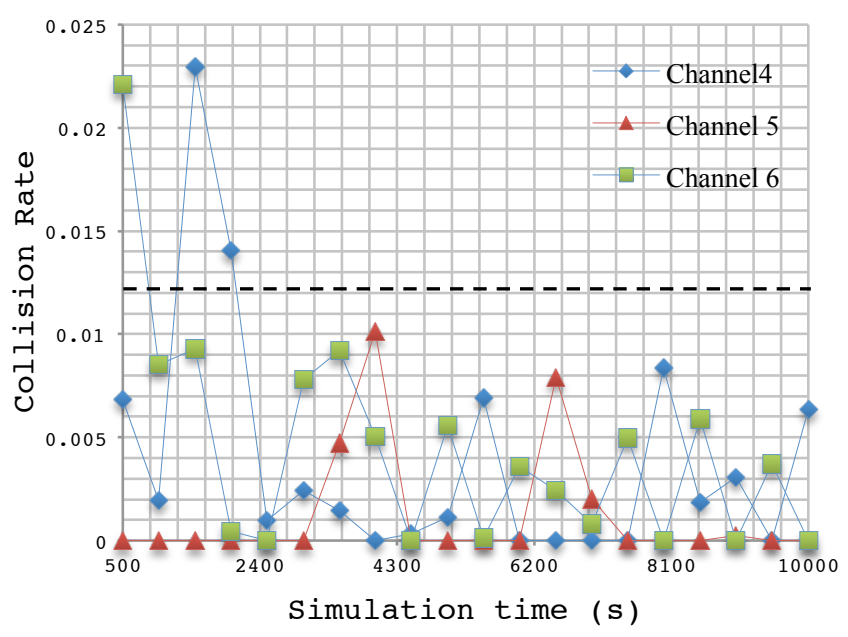

Figure 8. Channel collision control in licensed channels 4, 5, 6 during 10000s for ASOA scheme

\section{CONCLUSION AND FUTURE WORKS}

In this paper, we have considered a cognitive radio network located within a primary network with multi-licensed channels. On the basis of this model, we proposed a spectrum decision mechanism called Adaptive Spectrum Opportunity Access (ASOA) mechanism in order to improve secondary usage of unoccupied frequency bands and reducing frequency handoff rate. The numerical performance results of our proposed scheme are evaluated against Minimum Channel Rate Access (MICRL) scheme. The results have confirmed that Adaptive Spectrum Opportunity Access (ASOA) scheme could be a more viable approach in terms of channel collision, spectrum handoff rate and successful secondary data delivery when compared with MICRL scheme. The results also showed that for ASOA scheme, channel collision peaked at 0.01 due to the channel collision rate constraint. The channel handoff rates for the schemes under investigation peaked at $14.8 \%$ and $19.6 \%$ for the ASOA and MICRL schemes respectively in the licensed channels during a time period of $10000 \mathrm{~s}$. Hence, successful secondary data delivery averages at $43 \mathrm{MB}$ and $38 \mathrm{MB}$ through ASOA and MICRL schemes respectively.

Consequently, ASOA scheme can be viable spectrum decision mechanism in comparison with MICRL scheme. In realistic systems, there exist many cases which requires investigation, such as different primary channel access models and various idle channel states distributions which might be considered during the decision making process. Also cooperative decision scenario using different channel modeling needs to be investigated.

\section{APPENDIX}

From (3), average expected channel collision in channel i can be given as,

$$
\mathbb{E} \mathcal{C}^{i}=\int_{0}^{T_{s}^{i}} x f_{X}(x) d x=\left[\mathbb{E}^{i}(\mathrm{X})-\left(T_{s}^{i}+\mathbb{E}^{i}(\mathrm{X})\right) e^{-\mu_{o f f}^{i} T_{s}^{i}}\right]
$$

And the collision rate in renewal period is obtained by,

$$
\frac{\mathbb{E} C^{i}}{\mathbb{E}^{i}(\mathrm{X})+\mathbb{E}^{i}(\mathrm{Y})} \leq \delta^{i}
$$

Where $\delta^{i}$ represents channel collision constraint in channel i. Also the expression above can be rewritten as,

$$
\left[\mathbb{E}^{i}(\mathrm{X})-\left(T_{s}^{i}+\mathbb{E}^{i}(\mathrm{X})\right) e^{-\mu_{o f f}^{i} T_{s}^{i}}\right] \leq \delta^{i}\left(\mathbb{E}^{i}(\mathrm{X})+\mathbb{E}^{i}(\mathrm{Y})\right)
$$

By solving (17), $T_{s}^{i}$ is computed as,

$$
T_{s}^{i} \leq\left[\delta^{i}\left(\mathbb{E}^{i}(X)+\mathbb{E}^{i}(Y)\right) \cdot \mathbb{E}^{i}(X)\right]^{1 / 2}
$$

\section{REFERENCES}

[1] Wireless Technology and Spectrum Working Group, "Optimising use of available Mobile Radio Spectrum," Digital Communications Knowledge Transfer Network, 2011.

[2] "The spectrum impreative: Mobile broadband spectrum and its impacts for U.S. consumers and the economy," in Rysavy Research, 2011.

[3] "Estimated spectrum bandwidth requirements for the future development of IMT-2000 and IMT-Advanced," International Telecommunication Union, 2010.

[4] "Spectrum policy task force report," Federal Communications Comission, 2002.

[5] V. Valenta, R. Maršálek, G. Baudoin, M. Villegas, M. Suarez, and F. Robert, "Survey on spectrum utilization in Europe: Measurements, analyses and observations," in Fifth International Conference on Cognitive Radio Oriented Wireless Networks \& Communications (CROWNCOM 2010), 2010.

[6] Qing Zhao, Brian M. Sadler, "A Survey of dynamic spectrum access: Signal processing, networking, and regulatory policy," IEEE Signal Processing Magazine, no. 24, pp. 79-89, Mar. 2007.

[8] Simon Haykin, "Cognitive Radio: Brain-empowered 
wireless communications," IEEE Journal on Selected Area in communications, vol. 23, no. 2, pp. 201-220, Febuary 2005.

[7] J. Mitola III, "Cognitive radio: An integrated agent architecture for software defined radio," KTH Royal Institue of Technology, 2000.

[9] Qianchuan Zhao, Stefan Geirhofer, Lang Tong, Brian M. Sadler, "Opportunistic spectrum access via periodic channel sensing," IEEE Transaction on signal processing, no. 2, pp. 785-796, Feburary 36.

[10] Qing Zhao, Lang Tong, Ananthram Swami, and Yunxia Chen, "Decentralized cognitive MAC for opportunistic spectrum access in Ad Hoc networks: A POMDP framework," IEEE Journal on Selected Areas in Communications, vol. 56, no. 3, pp. 589-600, February 2007.

[11] Rui-Ting Ma, Yu-Pin Hsu, and Kai-Ten Feng, "A POMDP-based spectrum handoff protocol for partially observable cognitive radio networks," in IEEE Wireless Communications and Networking Conference (WCNC), 2009.

[12] Lei Yang, Lili Cao, Haitao Zheng, "Proactive channel access in dynamic spectrum networks," Physical communication, vol. 1, no. 2, pp. 103-111, 2008.

[13] Yi Song, Jiang Xie, "Common hopping based proactive spectrum handoff in cognitive radio Ad Hoc networks ," in IEEE Global Communications Conference (GLOBECOM), 2010.

[14] Xin Li, Qianchuan Zhao, Xiaohong Guan, Fellow, Lang Tong, "'Optimal Cognitive Access of Markovian Channels under Tight Collision Constraints," IEEE Journal on Selected Areas in Communications, vol. 29, no. 4, pp. 746-759, April 2011.

[15] Li-Chun Wang, Anderson Che, "On the performance of spectrum handoff for link maintenance in cognitive radio," in IEEE International Symposium on Wireless Pervasive Computing (ISWPC), 2008.

[16] Beibei Wang, Zhu Ji, K. J. Ray Liu, T. Charles Clancy, "Primary-Prioritized markov approach for dynamic spectrum allocation," IEEE Transactions on Wireless Communications, vol. 8, pp. 1854-1865, April 2009.

[18] Hsien-Po Shiang, Mihaela van der Schaar, "QueuingBased dynamic channel selection for heterogeneous multimedia applications over cognitive radio network," IEEE Transactions on Multimedia, vol. 10, no. 5, pp. 896-909, Augaust 2008.

[17] F. Borgonovo, M. Cesana, L. Fratt, "Throughput and delay bounds for cognitive transmissions," Advance in Ad Hoc Networking, vol. 265, pp. 179-190, August 2008.

[19] Ohyun Jo, Dong-Ho Cho, "Efficient spectrum matching based on spectrum characteristics in cognitive radio systems," in Wireless
Telecommunication Symposium, 2008, pp. 230-235.

[20] Zhigang Wen, Chunxiao Fan, Xiaoying Zhang, Yuexin $\mathrm{Wu}$, Junwei Zou, Jie Liu, "A learning spectrum hole prediction model for cognitive radio systems," in 10th IEEE International Conference on Computer and Information Technology, Bradford, UK, 2010.

[21] Ali Motamedi and Ahmad Bahai, "Optimal channel selection for spectrum- agile low- power wireless packet switched networks in unlicensed band," EURASIP Journal on Wireless Communications and Networking, vol. 2008, pp. 1-10, March 2008.

[22] Qinghai Xiao, Yunzhou Li, Ming Zhao, Shidong Zhou, Jing Wang, "Opportunistic channel selection approach under collision probability constraint in cognitive radio systems," Computer Communication, vol. 32, pp. 19141922, 2009.

[23] Suk-Un Yoon and Eylem Ekici, "Voluntary spectrum handpff: a novel approach to spectrum management in CRNs," in IEEE ICC 2010, 2010.

[24] Trivedi, Kishor Shridharbhai, Probability and Statistics with Reliability, Queuing, and Computer Science Applications, 2nd ed. New York: John Wiley \& Sons Ltd., 2002.

[25] Xiukui Li, Seyed Alireza Zekavat, "Traffic Pattern Prediction and Performance Investigation for Cognitive Radio Systems," in WCNC, 2008, pp. 894-899.

[26] Sheldon M.Ross, Stochatic Process, 2nd ed. New York: John Wiley \& Son, 1996.

[28] Mahdi Pirmoradian, Christos Politis, "Optimal Spectrum Hole Selection \& Exploitation in Cognitive Radio Networks," in Internationa Telecommunication Union- Kladoscopic (ITU-K2011), Cape Town, 2011.

[27] Mahdi Pirmoradian, Christos Politis, "Intelligent Channel Access Technique in Cognitive Radio Networks," in 10th IEEE Conference on Cybernetic and Intelligent Systems (CIS2011), London, 2011.

[29] H. C. Tjim, Stochastic Models An Algorithmic Approach. New York: John Wiley \& Sons Ltd, 1994.

[30] Manuj Sharma, Anirudha Sahoo, K. D. Nayak, "Traffic pattern prediction and performance investigation for cognitive radio systems," Dept. of Computer Science and Engineering, Indian Institute of Technology, Technical 2008. 